\title{
Síndrome de Achenbach de presentación inusual
}

\author{
Unusual presentation Achenbach syndrome
}

\author{
Yelson A. Picón-Jaimes ${ }^{*}$, Javier E. Orozco-Chinome², Mabel P. Franky-Rojas ${ }^{3}$, Jessica Molina-Franky', \\ Carlos Acosta-Guevara ${ }^{5}$ y Luis E. Echeverry²
}

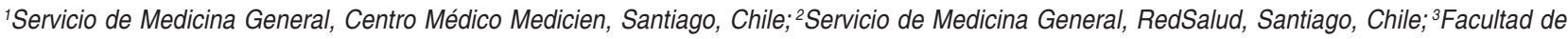
Ciencias de la Salud, Universidad de Boyacá, Tunja, Colombia; ${ }^{4}$ Facultad de Ciencias, Universidad Nacional de Colombia, Bogotá, Colombia; ${ }^{5}$ Servicio de Urgencias, Hospital Universitario San Ignacio, Colombia

\begin{abstract}
Resumen
El síndrome de Achenbach es una condición de etiología desconocida, caracterizado por cambios en la coloración de la piel de los dedos y asociado a dolor agudo. Existen pocos datos epidemiológicos, pero se estima que es una condición rara, que excepcionalmente aparece por debajo de los 40 años de edad. Presentamos el caso de una mujer joven a quien se dio el diagnóstico de síndrome de Achenbach por sus antecedentes y después de descartar patología reumática, vascular y metabólica. Comentamos datos sobre la patología y las diferencias que se encuentran con el caso que describimos.
\end{abstract}

Palabras clave: Hematoma. Enfermedades del pie. Equimosis. Piel. Anormalidades de la piel.

\begin{abstract}
Achenbach syndrome is a condition of unknown etiology, characterized by changes in the coloration of the skin of the fingers and associated with acute pain. There are few epidemiological data, but it is estimated that it is a rare condition, which exceptionally appears under 40 years of age. We present the case of a young woman who has been diagnosed with Achenbach syndrome thanks to her history and after ruling out rheumatic, vascular, and metabolic pathology. We finalize by discussing data on the pathology and the differences found with the case we describe here.
\end{abstract}

Key words: Hematoma. Foot diseases. Ecchymosis. Skin. Skin abnormalities.

\section{Introducción}

El síndrome de Achenbach, también conocido como hematoma paroxístico digital, es un trastorno que consiste en la aparición súbita de una coloración azul-violácea en la cara volar de los dedos de las manos, los dedos de los pies y raramente las palmas o las plantas ${ }^{1,2}$. Fue descrito por primera vez en la literatura médica en los años 1950 por el médico alemán Walter Achenbach, quien acuñó el epónimo por el cual se conoce a esta afección hasta la actualidad $^{2,3}$.

Se considera una patología infrecuente, poco reportada en la literatura, de curso benigno y autolimitada,

\footnotetext{
Correspondencia:

*Yelson A. Picón-Jaimes

La Gloria 47

Las Condes

Fecha de recepción: 30-10-2020

C.P. 7550000 , Santiago, Chile

E-mail: ypicon@unab.edu.co

0009-7411/@ 2021 Academia Mexicana de Cirugía. Publicado por Permanyer. Este es un artículo open access bajo la licencia CC BY-NC-ND (http://creativecommons.org/licenses/by-nc-nd/4.0/).
} 
que supone un reto para el personal clínico, pues suele confundirse con lesiones vasculares o con trastornos hematológicos o reumatológicos, haciendo difícil llegar a su diagnóstico inicialmente ${ }^{4,5}$.

La mayoría de los textos disponibles en las diferentes bases de datos corresponden a cartas al editor y solo unos pocos a reportes de casos. Por ello, se consideró importante realizar este manuscrito, pues este síndrome es una patología poco conocida por el personal de salud, que puede generar gran confusión en los servicios de urgencia y que requiere un alto grado de sospecha por parte del profesional de salud ${ }^{6,7}$.

Se considera que los síntomas presentados en el síndrome de Achenbach son secundarios al aumento de la resistencia capilar y la fragilidad vascular, que generan un hematoma que produce a su vez compresión y extravasación de sangre, diferenciándose de otras afecciones vasculares porque el recuento de plaquetas y los factores de la coagulación se encuentran en rango normal. En ausencia de una causa establecida o un tratamiento efectivo descrito, conocer la enfermedad permite ayudar a tranquilizar a los pacientes, ya que el curso es benigno y autolimitado?.

El objetivo de este artículo es presentar un caso inusual de síndrome de Achenbach y hacer una revisión de aspectos importantes sobre la patología.

\section{Caso clínico}

Mujer de 26 años con antecedente de hipotiroidismo en suplencia con levotiroxina $50 \mu \mathrm{g} / \mathrm{día}$, que consulta por lesión en la planta del pie derecho tipo equimosis de aproximadamente $3 \times 2 \mathrm{~cm}$, de aparición súbita mientras realizaba una sesión de yoga (Fig. 1). Refiere dolor agudo tipo punzada y la consecuente visualización de la lesión descrita, por lo cual decidió consultar para evaluación médica. A su ingreso muestra presión arterial de 117/75 mmHg, frecuencia cardiaca de 78 latidos por minuto, frecuencia respiratoria de 16 respiraciones por minuto, saturación de oxígeno al aire ambiente del $98 \%$ y temperatura axilar de $36.7^{\circ} \mathrm{C}$. En la exploración segmentaria se evidenció equimosis en la planta del pie derecho, no dolorosa a la palpación, con temperatura local conservada, buen llenado capilar distal y pulsos periféricos (pedio y tibial posterior) presentes. Al interrogar a la paciente, manifestó que esto le había sucedido en dos ocasiones anteriores, la primera vez en la mano izquierda mientras se encontraba en su trabajo digitando en el computador, con resolución espontánea a las 2 semanas sin ningún tratamiento, y la segunda vez en el pie derecho durante una jornada

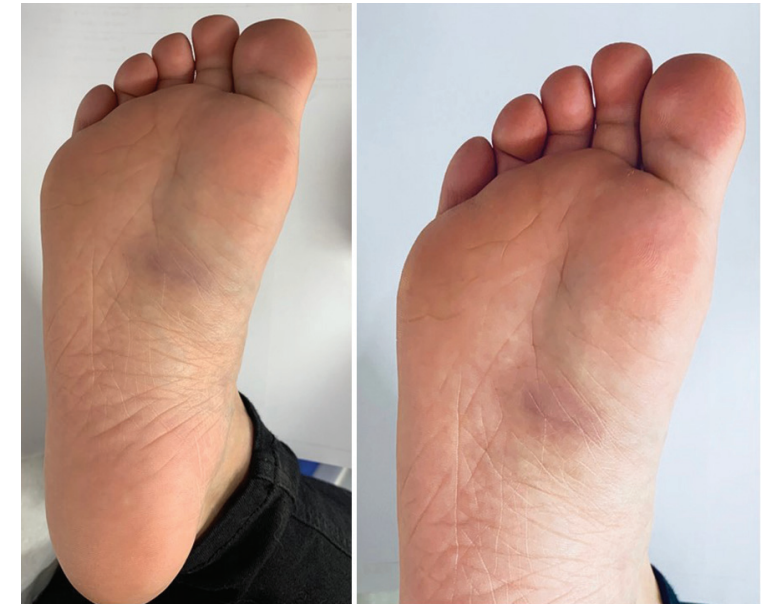

Figura 1. Fotografía que muestra la lesión tipo equimosis.

deportiva; en esa ocasión lo consideró como una lesión por trauma secundario al deporte, por lo cual no solicitó atención médica. Debido a la naturaleza repetitiva de la lesión, se explicó a la paciente que era necesario descartar trastornos hematológicos, por lo cual se solicitaron laboratorios, que reportaron bilirrubina total de $0.58 \mathrm{mg} / \mathrm{dl}$, transaminasa oxalacética $16 \mathrm{U} / \mathrm{l}$, transaminasa pirúvica $14 \mathrm{U} / \mathrm{l}$, gamma-glutamil transpeptidasa $5 \mathrm{U} / \mathrm{l}$, fosfatasa alcalina $40 \mathrm{U} / \mathrm{l}$, protrombina con producción al 100\%, INR 1.0, proteína C reactiva $0.3 \mathrm{mg} / \mathrm{dl}$, hormona estimulante de tiroides $1.53 \mathrm{ulU} / \mathrm{ml}$, hemoglobina $12.7 \mathrm{~g} / \mathrm{dl}$, recuento de leucocitos $9000 / \mathrm{ml}$, plaquetas $385,000 / \mathrm{ml}$, velocidad de sedimentación $10 \mathrm{~mm} / \mathrm{h}$, creatina cinasa total $48 \mathrm{U} / \mathrm{l}$, creatinina $0.68 \mathrm{mg} / \mathrm{dl}$, glucemia basal $75 \mathrm{mg} / \mathrm{dl}$, anticuerpos anticitoplasma de neutrófilos negativo, anticuerpos antinucleares negativo (patrones nucleares negativo/patrones citoplasmáticos o mitóticos negativo), antiestreptolisina $<25.0 \mathrm{Ul} / \mathrm{ml}$, factor reumatoide negativo y péptido citrulinado-anticuerpos IgG $1.60 \mathrm{RU} / \mathrm{ml}$ (negativo). Adicionalmente, se realizaron una radiografía (Fig. 2), que evidenció un leve hallux valgus, sin signos de lesión ósea traumática, y una ecotomografía que dio cuenta de partes blandas visibles sin alteraciones. Por lo anterior, y luego de descartar una lesión ósea, vascular o reumatológica, se consideró el diagnóstico de síndrome de Achenbach de presentación inusual por la edad de la paciente y la localización del evento.

\section{Discusión y conclusiones}

El síndrome de Achenbach se define como la aparición de hematomas en los dedos o las palmas, pero 


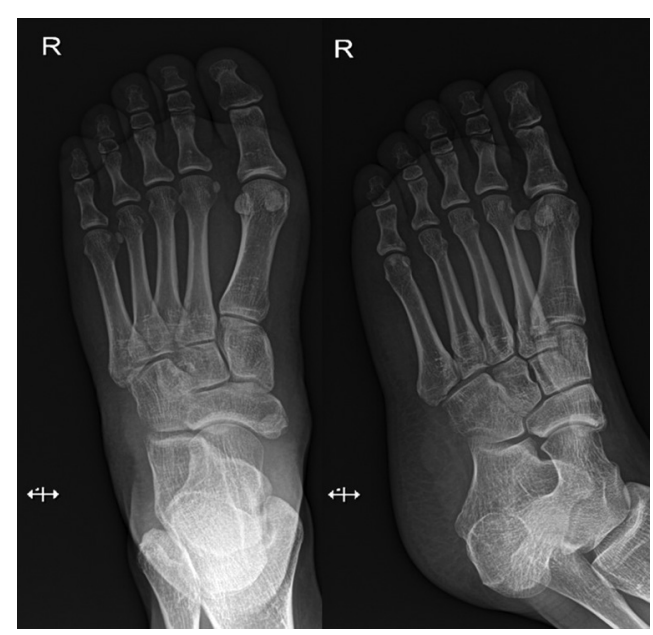

Figura 2. Radiografía del pie derecho de la paciente que da cuenta de la integridad ósea.

dentro del espectro caben otros tipos de lesiones, como las equimosis e incluso las púrpuras ${ }^{5}$. Es importante señalar que no hay cambios con respecto a la exposición al frío y el llenado capilar se mantiene normal ${ }^{8}$, lo cual es similar a lo encontrado en nuestro caso.

En cuanto a la presentación respecto al sexo, se ha evidenciado que es más frecuente en las mujeres, con una relación 7:1 frente a los hombres. En general aparece en personas mayores de 50 años ${ }^{5,9}$, situación que dista de la realidad de nuestra paciente, cuya edad es inferior a los 30 años, y concuerda con el caso reportado por Suzuki ${ }^{10}$ en 2019 , de una mujer de 30 años, de Japón, que debutó con este trastorno acompañado de dolor y fiebre, con analítica sanguínea normal.

Otros síntomas comunes son el edema y el dolor en la zona afectada, los cuales están presentes en aproximadamente el $50-60 \%$ de los casos. Las parestesias pueden aparecer incluso hasta en un $25 \%$ de los episodios ${ }^{11}$ y un hallazgo excepcional que ha sido reportado es la hemorragia subconjuntival ${ }^{12}$, en una paciente que presentó dolor agudo y de gran intensidad; sin embargo, otros autores también han descrito la presencia de una sensación urente o pulsátil acompañando a este trastorno ${ }^{13,14}$.

El diagnóstico es plenamente clínico, pero como ya se mencionó, la intensidad del dolor lleva a estos pacientes a acudir al sistema de emergencias, donde una anamnesis exhaustiva y una buena exploración fisica son suficientes para orientar el reconocimiento de la patología $a^{15}$. Los principales diagnósticos diferenciales son los trastornos de la coagulación, las enfermedades reumatológicas y los trastornos vasculares. Por lo tanto, datos físicos como el dolor, y la persistencia de la temperatura normal, lo diferencian de patologías como la acrocianosis, en la cual la piel se torna fría y no hay dolor, y del síndrome de Reynaud, que empeora con el frío y muestra cambios tróficos ${ }^{16,17}$. Ayudas diagnósticas como la radiografía son importantes para descartar alteraciones óseas, ya que muchas veces el síndrome de Achenbach aparece durante la realización de actividad física. La ecotomografía Doppler es eficaz para descartar compromiso circulatorio distal de la extremidad, y la analítica sanguínea determina la normalidad en las pruebas de coagulación, e incluso permiten determinar la presencia de alteraciones reumatológicas ${ }^{18,19}$.

El tratamiento suele ser de soporte, debido al curso benigno y autolimitado del síndrome. Una vez orientado el diagnóstico, y descartadas patologías de otra índole, debe explicarse al paciente la evolución natural del trastorno, pues se ha evidenciado que es recidivante, aunque no se conocen con exactitud las situaciones desencadenantes o predisponentes. El uso de analgésicos y antiinflamatorios no esteroideos suele ser suficiente para manejar el dolor y el edema ${ }^{15,20}$.

Finalmente, consideramos que el nombre del trastorno debe ser hematoma paroxístico acral, como proponen Pavlović y Loubser ${ }^{7}$, pues puede afectar otras partes de las palmas y las plantas de los pies, y no simplemente estar limitado a los dedos.

\section{Responsabilidades éticas}

Protección de personas y animales. Los autores declaran que los procedimientos seguidos se conformaron a las normas éticas del comité de experimentación humana responsable y de acuerdo con la Asociación Médica Mundial y la Declaración de Helsinki.

Confidencialidad de los datos. Los autores declaran que han seguido los protocolos de su centro de trabajo sobre la publicación de datos de pacientes.

Derecho a la privacidad y consentimiento informado. Los autores han obtenido el consentimiento informado de los pacientes y/o sujetos referidos en el artículo. Este documento obra en poder del autor de correspondencia.

\section{Contribución de los autores}

La totalidad de los autores participaron activamente en la discusión de los resultados, la revisión y la aprobación de la versión final del trabajo. 


\section{Financiación}

Los autores declaran que no existen fuentes de financiación externa para el presente artículo.

\section{Conflicto de intereses}

Los autores declaran no tener ningún conflicto de intereses.

\section{Bibliografía}

1. Frerix M, Richter K, Müller-Ladner U, Hermann W. Achenbach's syndrome (paroxysmal finger hematoma) with capillaroscopic evidence of microhemorrhages. Arthritis Rheumatol. 2015;67:1073.

2. Kordzadeh $A$, Caine $P$, Jonas $A$, Rhodes $K$, Panayiotopolous $Y$. Is Achenbach's syndrome a surgical emergency? A systematic review. Eur J Trauma Emerg Surg. 2016;42:439-43.

3. Castillo S, Dinulos J, Werchniak A. Painful ecchymosis of the finger: a case of Achenbach syndrome. Int J Dermatol. 2019;59:862-3.

4. Sigha B, Josselin L, Gatfosse M, Fardet L. Hématomes digitaux spontanés paroxystiques (syndrome d'Achenbach). Ann Dermatol Venereol. 2016;143:130-3. Disponible en: https://linkinghub.elsevier.com/retrieve/ pii/S0151963815012314

5. Restrepo Jiménez P, Ocampo MI, Castañeda-Cardona C, Rosselli D. Síndrome de Achenbach: presentación de un caso y revisión sistemática de la literatura. Rev Colomb Reumatol. 2017;24:230-6.
6. Watchorn R, Babu S, Lewis F, Calonje E, Taibjee S. Paroxysmal purple palmar macules with a rare aetiology. Clin Exp Dermatol. 2017;42:561-3.

7. Pavlović M, Loubser M. Paroxysmal acral haematoma is a more appropriate name for Achenbach syndrome. Clin Exp Dermatol. 2019;44:e18-9.

8. Yamamoto $\mathrm{Y}$, Yamamoto $\mathrm{S}$. Achenbach's syndrome. N Engl J Med. 2017;376:e53.

9. Seifman M, Johnstone B. The acutely blue finger: cause for concern? J Hand Surg (Asian-Pacific). 2018;23:294-6.

10. Suzuki J. Achenbach's syndrome in a 30 -year-old healthy woman. Intern Med. 2019;58:1807.

11. Yamada T. Achenbach's syndrome in an elderly woman. J Gen Fam Med. 2018;19:65-6.

12. Young B, Okera S. Recurrent subconjunctival haemorrhage in Achenbach's syndrome. Clin Exp Ophthalmol. 2018;46:965-6.

13. Ahmed Z, Elmallah A, Elnagar M, Dowdall J, Barry M, Sheehan S. Painful blue finger - Achenbach's syndrome: two case reports. EJVES Short Reports. 2018;40:1-2.

14. Cohen SN. Personal observations of Achenbach syndrome. Clin Exp Dermatol. 2018;43:933-4.

15. Picón Jaimes Y, Orozco Chinome J, Molina-Franky J. Hematoma digital espontáneo, síndrome de Achenbach. Rev Fac Cienc Med Cordoba. 2019;76:257-60.

16. Yie K. Achenbach syndrome: a benign painful blue finger with tip sparing. Vasc Spec Int. 2019;35:251-5.

17. Ada F, Kasimzade F. Analysis of 24 patients with Achenbach's syndrome. World J Clin Cases. 2019;7(10):1103-1110.

18. Godoy A, Tabares AH. Achenbach syndrome (paroxysmal finger hematoma). Vasc Med. 2019;24:361-6. Disponible en: http://journals.sagepub. com/doi/10.1177/1358863X19849627

19. Notomi K, Harada T. Achenbach syndrome. CMAJ. 2019;191:E584.

20. Ribeiro F, Aveiro M, Leal M, Valente T, Jesus G. An acute blue finger: a case of Achenbach's syndrome. Eur J Case Reports Intern Med. 2019;6:9-11. 